\title{
DISCOVERY OF A T DWARF BINARY WITH THE LARGEST KNOWN $J$-BAND FLUX REVERSAL ${ }^{1}$ \\ Dagny L. Looper, ${ }^{2}$ Christopher R. Gelino, ${ }^{3}$ Adam J. Burgasser, ${ }^{4}$ and J. Davy Kirkpatrick ${ }^{5}$ \\ Received 2008 February 29; accepted 2008 May 19
}

\begin{abstract}
We present Keck laser guide star observations of two T2.5 dwarfs - 2MASS J11061197+2754225 and 2MASS J14044941-3159329 - using NIRC2 on Keck II and find 2MASS J14044941-3159329 to be a $0.13^{\prime \prime}$ binary. This system has a secondary that is 0.45 mag brighter than the primary in $J$ band, but 0.49 mag fainter in $H$ band and 1.13 mag fainter in $K_{s}$ band. We use this relative photometry along with near-infrared synthetic modeling performed on the integrated light spectrum to derive component types of $\mathrm{T} 1 \pm 1$ for the primary and $\mathrm{T} 5 \pm 1$ for the secondary. Optical spectroscopy of this system obtained with Magellan/LDSS-3 is also presented. This is the fourth $\mathrm{L} / \mathrm{T}$ transition binary to show a flux reversal in the $1-1.2 \mu \mathrm{m}$ regime, and this one has the largest flux reversal. Unless the secondary is itself an unresolved binary, the $J$-band magnitude difference between the secondary and primary shows that the $J$-band "bump" is indeed a real feature and not an artifact caused by unresolved binarity.
\end{abstract}

Subject headings: binaries: close — binaries: general — stars: individual (2MASS J11061197+2754225, 2MASS J14044941-3159329) — stars: low-mass, brown dwarfs — techniques: high angular resolution — techniques: spectroscopic

Online material: color figures

\section{INTRODUCTION}

As brown dwarfs cool and pass through the L/T spectral class boundary, their spectral morphologies transition from red nearinfrared (NIR) colors of the L dwarf class, caused by condensate dust in their photospheres, to blue NIR colors of the T dwarf class, where their photospheres are relatively clear of dust. This transition is rapid, as implied by the nearly flat effective temperature scale around $1400 \mathrm{~K}$ for NIR L7-T5 dwarfs (Golimowski et al. 2004; Kirkpatrick 2005, bottom panel of Fig. 7 in that paper). Within this transition, a remarkable brightening in $J$ band $\left(\Delta M_{J} \sim 1\right)$ from spectral types $\sim \mathrm{T} 1-\mathrm{T} 5$, known as the $J$-band "bump" (Dahn et al. 2002; Tinney et al. 2003; Vrba et al. 2004), has been noted.

Two withstanding mechanisms to explain this brightening have been suggested: (1) the "patchy clouds" model-proposed by Burgasser et al. (2002; see also Ackerman \& Marley 2001), suggesting that the breakup of clouds in the atmosphere allows hot flux from inner layers to emerge (analogous to the $5 \mu \mathrm{m}$ hot spots of Jupiter); and (2) the "sudden downpour" modelproposed by Knapp et al. (2004), suggesting that the dust clouds suddenly condense out due to an increase in sedimentation efficiency.

\footnotetext{
${ }^{1}$ Some of the data presented herein were obtained at the W. M. Keck Observatory, which is operated as a scientific partnership among the California Institute of Technology, the University of California, and the National Aeronautics and Space Administration. The Observatory was made possible by the generous financial support of the W. M. Keck Foundation. This paper includes data gathered with the $6.5 \mathrm{~m}$ Magellan Telescopes located at Las Campanas Observatory, Chile.

2 Institute for Astronomy, University of Hawaii, 2680 Woodlawn Drive, Honolulu, HI 96822.

${ }^{3}$ Spitzer Science Center, MS 220-6, California Institute of Technology, Pasadena, CA 91125.

${ }^{4}$ MIT Kavli Institute for Astrophysics and Space Research, 77 Massachusetts Avenue, Building 37-664B, Cambridge, MA 02139.

5 Infrared Processing and Analysis Center, MS 100-22, California Institute of Technology, Pasadena, CA 91125.
}

Recent studies of brown dwarf binaries have revealed that a fraction of the amplitude in this bump can be explained by systems appearing overluminous due to binarity (i.e., "cryptobinarity"; Burgasser et al. 2006c; Liu et al. 2006; Burrows et al. 2006); however, some part of this brightening is intrinsic to the atmospheres as they cool. This was revealed by Hubble Space Telescope (HST) Wide Field Planetary Camera 2 imaging of 2MASS J17281150+3948593AB (hereafter 2MASS 1728AB), the first $1-1.2 \mu \mathrm{m}$ flux reversal binary, which has a $\mathrm{T}$ dwarf secondary brighter in $z$ band but fainter in $i$ band than the mid- to latetype L dwarf primary (Gizis et al. 2003). No $J$-band resolved photometry for this system has been published. Two later discoveries of 1-1.2 $\mu \mathrm{m}$ flux reversal binaries - SDSS J102109.69030420.1 (hereafter SDSS 1021; HST Near-Infrared Camera and Multi-Object Spectrometer; Burgasser et al. 2006c) and SDSS J153417.05+161546.1 (hereafter SDSS 1534; Keck LGS AO/ NIRC2; Liu et al. 2006) - provided additional information on the flux reversals. Both systems were found to have a secondary brighter than the primary in $J$ band (see Table 1).

$\mathrm{L}$ dwarf/T dwarf binaries such as those listed above provide crucial information on the $\mathrm{L} / \mathrm{T}$ transition, as the components of these systems are likely coeval, with common ages and compositions. In an effort to identify additional systems, we have performed high angular resolution imaging of two T2.5 dwarfs - 2MASS J11061197+2754225 (hereafter 2MASS 1106) and 2MASS J14044941-3159329 (hereafter 2MASS 1404). These objects were identified in a NIR proper-motion survey (Looper et al. 2007; J. D. Kirkpatrick et al. 2008, in preparation) based on multiepoch data from the Two Micron All Sky Survey (2MASS; Skrutskie et al. 2006). Both systems are in the field, unassociated with any higher mass stars within a radius of $10^{\prime} .^{6}$ 2MASS 1404 was discovered to be a fourth resolved L/T transition system showing this $1-1.2 \mu \mathrm{m}$ flux reversal. These observations were made using the Keck II sodium laser guide star adaptive optics system.

\footnotetext{
${ }^{6}$ See http://simbad.u-strasbg.fr/simbad/.
} 
TABLE 1

$J$-Band Flux Reversal Binaries

\begin{tabular}{|c|c|c|c|c|c|c|c|}
\hline Designation & $J^{2 \text { MASS }}$ & $\Delta J_{\mathrm{A}-\mathrm{B}}^{2 \mathrm{MASSa}}$ & $\Delta J_{\mathrm{A}-\mathrm{B}}^{\mathrm{MKOb}}$ & $\mathrm{SpT} \mathrm{AB}^{\mathrm{c}}$ & $\mathrm{SpT} \mathrm{A}^{\mathrm{c}}$ & $\mathrm{SpT} \mathrm{B}^{\mathrm{c}}$ & Reference \\
\hline 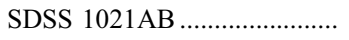 & $16.25 \pm 0.09$ & -0.05 & 0.04 & $\mathrm{~T} 3$ & $\mathrm{~T} 1$ & $\mathrm{~T} 5$ & 1 \\
\hline 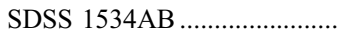 & $16.75 \pm 0.13$ & 0.03 & 0.17 & $\mathrm{~T} 3.5$ & $\mathrm{~T} 1.5$ & T5.5 & 2 \\
\hline 2MASS 1404AB ..................... & $15.56 \pm 0.09$ & 0.45 & 0.53 & $\mathrm{~T} 2.5$ & $\mathrm{~T} 1$ & T5 & 3 \\
\hline
\end{tabular}

NotE.-There is one other (possible) $J$-band flux reversal binary not tabulated here: 2MASS $1728 \mathrm{AB}$ because it has been observed at $z$ band, not at $J$ band (see $\S 1)$.

a Relative 2 MASS photometry calculated in this paper.

b Relative MKO photometry from references.

c NIR spectral types on Burgasser et al. (2006b) scheme.

REFERENCES.-(1) Burgasser et al. 2006c; (2) Liu et al. 2006; (3) this paper.

We describe these observations and optical spectroscopy of 2MASS 1404 in $\S 2$, discuss the results of this imaging and implications for the $J$-band bump in $\S 3$, and give our conclusions in $\S 4$.

\section{OBSERVATIONS}

\subsection{High-Resolution Imaging: Keck II $10.0 \mathrm{~m} / N I R C 2$ LGS AO}

We used the Keck II 10.0 m Telescope ${ }^{7}$ Sodium Laser Guide Star Adaptive Optics (LGS AO) system (Wizinowich et al. 2006; van Dam et al. 2006) on 2006 June 3 UT to observe 2MASS 1106 and 2MASS 1404. Observations were taken using the Near-Infrared Camera 2 (NIRC2) narrow camera and the Mauna Kea Observatories (MKO) filter set (Tokunaga et al. 2002): $K_{s}$ band (2.15 $\mu \mathrm{m}$ ) for 2MASS 1106 ; and $J(1.25 \mu \mathrm{m}), H(1.635 \mu \mathrm{m})$, and $K_{s}$ bands for 2MASS 1404. The field of view was $10.2^{\prime \prime}$, with a $0.00994^{\prime \prime}$ pixel scale. Nearby $\left(<60^{\prime \prime}\right)$ and bright $(R<19 \mathrm{mag})$ stars were selected from the USNO-B1.0 catalog (Monet et al. 2003) to provide for tip-tilt (TT) sensing. For 2MASS 1106, the TT star (USNOB1.0 1179-0233699) had a brightness of $R=15.2$ mag and a separation of $\sim 52^{\prime \prime}$. For 2MASS 1404, the TT star (USNO-B1.0 0580-0365843) had a brightness of $R=16.3 \mathrm{mag}$ and a separation of $\sim 29^{\prime \prime}$. Conditions were clear and photometric, with good seeing at the start of the night $\left(0.7^{\prime \prime}\right.$ at $K$ band) with slight degradation afterward.

For 2MASS 1106, we dithered by a few arcseconds between positions to obtain a set of eight images with an integration time of $60 \mathrm{~s}$ for each position, for a total on-source integration time of 480 s. For 2MASS 1404, we dithered by a few arcseconds between positions, for a total of six images for each of the three filters. Integration times per position were 90,60 , and $60 \mathrm{~s}$, for a total integration time of 540,360 , and $360 \mathrm{~s}$ in the $J, H$, and $K_{s}$ filters, respectively. The full widths at half-maximum (FWHMs) in the $K_{S}$ filter were $0.068^{\prime \prime}$ and $0.065^{\prime \prime}$ for 2MASS 1106 and 2MASS 1404, respectively.

We employed standard reduction techniques. Flats were created from differenced lights-on and lights-off images of the telescope dome interior. A super-sky frame was created from the median of the individual science frames and was subsequently subtracted from each frame. The background-subtracted images were shifted so that the target landed on the same position and were then stacked to form the final mosaic.

In the mosaic of 2MASS 1106 (see Fig. 1), the target is not elongated and no other objects, companions or background sources, are seen. On the other hand, we have resolved 2MASS 1404 into two components, shown in Figure 2. Based on the large proper motion of this system $\left(\mu=0.35 \operatorname{arcsec}^{-1}\right)$ and the elapsed $5.3 \mathrm{yr}$ between the first 2MASS observation of this field (2001 Feb-

\footnotetext{
7 Located on the summit of Mauna Kea, Hawaii.
}

ruary 4 UT) and our observation with Keck II (2006 June 3 UT), we should be able to see both components separated in the 2MASS image, which has a plate scale of $\sim 1^{\prime \prime}$, if they were not physically associated. Because we do not see an object on the 2001 2MASS image that is positionally coincident with the double seen in our 2006 Keck image, we conclude that this is a physical binary unresolved in 2MASS images. Using the $K_{s}$-band final mosaic, which has the smallest FWHM, we find a separation, $\rho$, of $0.1336^{\prime \prime} \pm 0.0006^{\prime \prime}$ and a position angle, $\phi$, of $311.8^{\circ} \pm 0.7^{\circ}$.

We performed an image subtraction technique in all three bands to obtain final flux ratios for the 2MASS $1404 \mathrm{AB}$ system. To do this, we computed the centroid for the component to be subtracted and placed this component at the center of a $257 \times 257$ pixel subimage. This subimage was copied, rotated $180^{\circ}$, and then subtracted from the original, nonrotated subimage. The quality of the subtraction was checked both visually and by examination of the radial profile of the unsubtracted component. In many cases, small offsets had to be applied by hand to obtain optimal subtraction. Aperture photometry was performed on the unsubtracted objects and used to obtain the flux ratio difference of the components.

Errors in the rotation subtraction technique were estimated by creating fake binaries from (presumably) single objects observed with NIRC2 in LGS AO mode and having similar image qualities as $2 \mathrm{MASS} 1404 \mathrm{AB}$. The fake binaries were created at 25 different separations and position angles. The same rotation subtraction

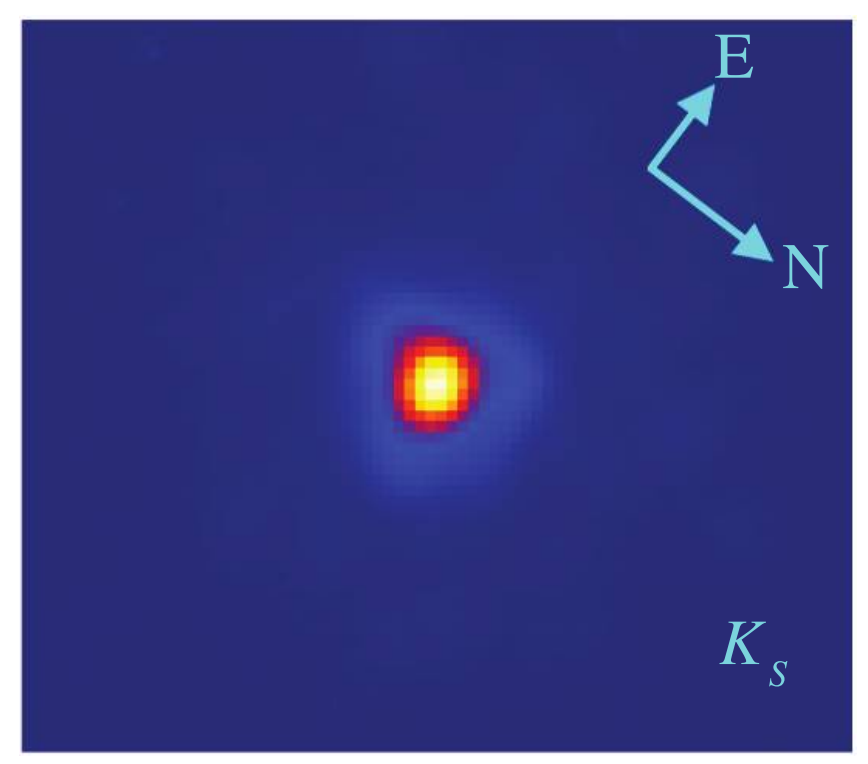

FIG. 1.-Keck LGS AO image of 2MASS 1106 in $K_{s}$ band. North and east are indicated by arrows. The image is $\sim 0.8^{\prime \prime}$ on a side, with no other sources besides the target detected in the full-sized $\left(\sim 13.5^{\prime \prime}\right)$ field of view. The FWHM is $0.068^{\prime \prime}$. 

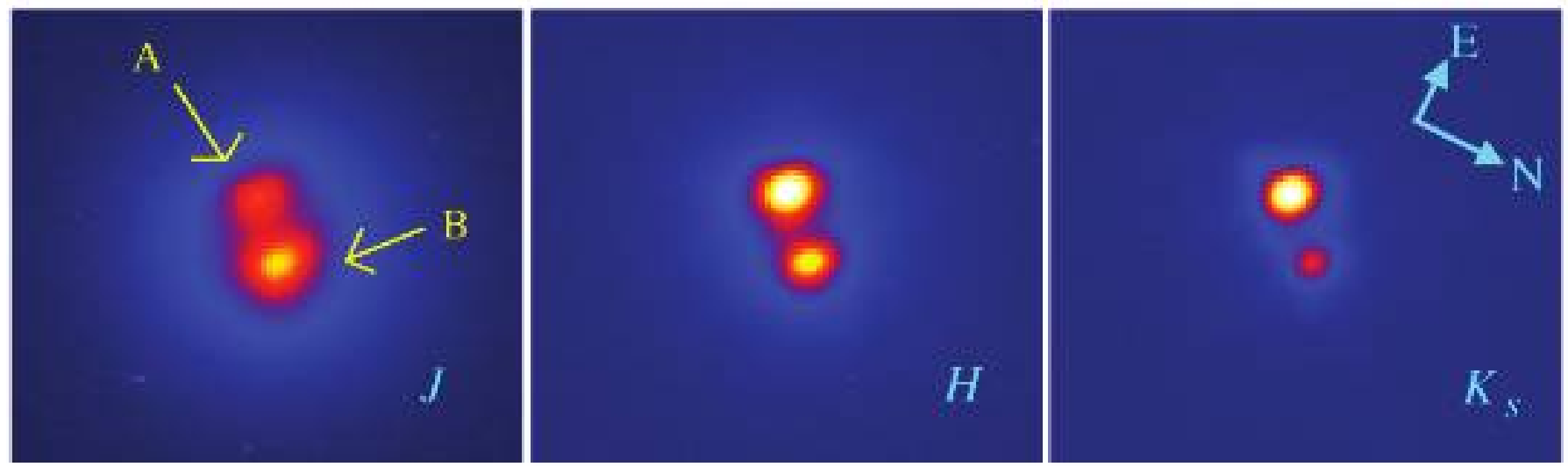

FIG. 2.-Keck LGS AO images of $2 \mathrm{MASS} 1404 \mathrm{AB}$ in $J, H$, and $K_{s}$ bands. North and east are indicated by arrows and are the same for all images. Note that the B component is brighter in $J$ band than the A component, but is fainter in both $H$ and $K_{s}$ bands. Each subimage is $\sim 0.8^{\prime \prime}$ on a side, and the separation of the binary is $0.1336^{\prime \prime} \pm 0.0006^{\prime \prime}$ with a position angle of $311.8^{\circ} \pm 0.7^{\circ}$. No other sources besides components A and B were detected in the full-sized $\left(\sim 14^{\prime \prime}\right)$ field of view. The FWHM is $0.065^{\prime \prime}$ in $K_{s}$ band.

technique was performed on these binaries as was done for 2MASS 1404AB. The magnitude of each component was taken to be the average of the 25 separate measurements, and the error was the standard deviation of the measurements.

The final errors in the photometry for 2MASS $1404 \mathrm{AB}$ are the square root of the sum of squares for the following eight terms: the errors in the single point-spread function (PSF) photometry (both A and B), the standard deviation of the binary PSF photometry (both $\mathrm{A}$ and $\mathrm{B}$ ), the difference between the single and binary PSF photometry (both A and B), and the errors in 2MASS 1404AB photometry (both A and B). The final flux ratios for 2MASS $1404 \mathrm{AB}$ are shown in Table 2.

TABLE 2

Properties of the 2MASS 1404AB SyStem

\begin{tabular}{|c|c|}
\hline Property & Value \\
\hline MKO $J(\mathrm{mag}) \ldots$ & $15.41 \pm 0.07$ \\
\hline MKO $H(\mathrm{mag}) .$. & $15.02 \pm 0.08$ \\
\hline MKO $K(\mathrm{mag}) \ldots .$. & $14.55 \pm 0.10$ \\
\hline MKO $\Delta J(\mathrm{mag}) \ldots$ & $0.53 \pm 0.16$ \\
\hline $\mathrm{MKO} \Delta H(\mathrm{mag}) . . . . . . . . .$. & $-0.48 \pm 0.11$ \\
\hline MKO $\Delta K(\mathrm{mag}) \ldots \ldots \ldots . . .$. & $-1.20 \pm 0.21$ \\
\hline 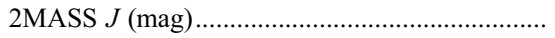 & $15.58 \pm 0.07$ \\
\hline 2MASS $H(\mathrm{mag}) \ldots \ldots \ldots \ldots . . . .$. & $14.96 \pm 0.08$ \\
\hline 2 MASS $K_{s}(\mathrm{mag}) \ldots \ldots \ldots \ldots \ldots$ & $14.54 \pm 0.10$ \\
\hline $2 \operatorname{MASS} \Delta J(\mathrm{mag}) \ldots \ldots \ldots \ldots \ldots . . . . . .$. & $0.45 \pm 0.15$ \\
\hline 2MASS $\Delta H$ (mag) & $-0.49 \pm 0.13$ \\
\hline 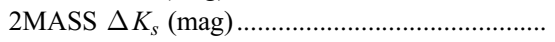 & $-1.13 \pm 0.22$ \\
\hline 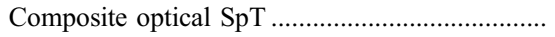 & $\mathrm{T} 0$ \\
\hline 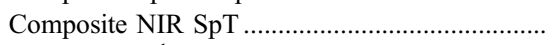 & $\mathrm{T} 2.5^{\mathrm{a}}$ \\
\hline 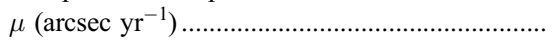 & $0.35 \pm 0.03^{\mathrm{a}}$ \\
\hline$\theta(\mathrm{deg})$ & $275.3 \pm 0.2^{\mathrm{a}}$ \\
\hline$\rho$ (mas) & $133.6 \pm 0.6$ \\
\hline$\phi(\operatorname{deg}) \ldots \ldots \ldots$ & $311.8 \pm 0.7$ \\
\hline $\log \left(L_{\mathrm{A}} / L_{\mathrm{B}}\right)^{\mathrm{b}} \ldots \ldots$ & $0.25 \pm 0.13$ \\
\hline 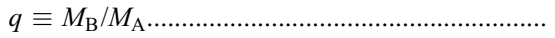 & $0.80 \pm 0.09$ \\
\hline$M_{\text {tot }}\left(M_{\text {Jup }}\right)$ for $0.5,1.0$, and $5.0 \mathrm{Gyr} \ldots \ldots \ldots \ldots \ldots . . . . . . . .$. & 50,70 , and 80 \\
\hline Est. distance $(\mathrm{pc})$ & $\sim 23$ \\
\hline Est. projected separation (AU) & $\sim 3.1$ \\
\hline 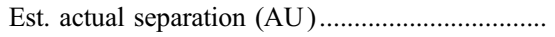 & $\sim 3.9$ \\
\hline 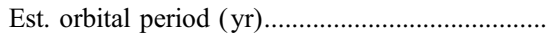 & $28-35$ \\
\hline
\end{tabular}

\footnotetext{
${ }^{\mathrm{a}}$ Measurements are from Looper et al. (2007).

b $\left(L_{\mathrm{bol}} / L_{\mathrm{bol} \odot}\right)=100^{\left(M_{\mathrm{bol} \odot}-M_{\mathrm{bol}}\right) / 5}$, where $M_{\mathrm{bol} \odot}=+4.76$.
}

\subsection{Red Optical Spectroscopy: Magellan $6.5 \mathrm{~m} /$ LDSS3}

Red optical spectroscopy ( $6000-10500 \AA)$ of the 2MASS $1404 \mathrm{AB}$ system was obtained on 2006 May 8 (UT) with the Low Dispersion Survey Spectrograph 3 (LDSS-3; upgraded from LDSS-2; Allington-Smith et al. 1994) on the $6.5 \mathrm{~m}$ Magellan Clay Telescope. Conditions were clear with good seeing $\left(\sim 0.6^{\prime \prime}\right.$ at $R$ band). The VPH-red grism (660 lines $\mathrm{mm}^{-1}$ ) and the OG590 longpass filter were used with a $0.75^{\prime \prime}$ (4 pixels) slit, resulting in $R=\lambda / \Delta \lambda \approx 1800$. The slit was rotated to the parallactic angle to minimize slit losses. Dispersion across the chip was $1.2 \AA$ pixel $^{-1}$. We obtained two exposures of $1500 \mathrm{~s}$ each, for a total integration time of 50 minutes at an average air mass of 1.01. The flux standard star LTT 7987 (Hamuy et al. 1994) was observed the previous night (2006 May 7 UT) using an identical setup. Calibration exposures were taken using the HeNeAr arc lamp and the flat-field quartz lamp. The G2 V star HD 127526 was observed immediately prior to $2 \mathrm{MASS} 1404 \mathrm{AB}$ to use as a correction for telluric absorption.

LDSS- 3 data were reduced in the IRAF ${ }^{8}$ environment. Raw science images were first trimmed, bias subtracted, and then divided by the normalized, median combined, and bias subtracted set of flat-field frames. Spectra were optimally extracted using the APALL task, with the extraction of HD 127526 used as a dispersion template for 2MASS 1404AB. Wavelength solutions were determined from the arc lamp spectra; solutions were accurate to $\sim 0.1$ pixels, or $\sim 0.12 \AA$. Flux calibration was determined using the tasks STANDARD and SENSFUNC with observations of LTT 7987, adequate over the spectral range 6000-10000 $\AA$. Corrections to telluric $\mathrm{O}_{2}(6860-6960 \AA$, B band; $7580-7700 \AA$, A band) and $\mathrm{H}_{2} \mathrm{O}$ (7150-7300 and 9270-9675 $\AA$ ) absorption bands were computed using the $\mathrm{G}$ dwarf spectrum.

The optical spectrum is shown in Figure 3, along with the L8 optical standard 2MASS J1632291+190441 (hereafter 2MASS 1632; Kirkpatrick et al. 1999) and the T2 optical standard SDSS J125453.90-012247.4 (hereafter SDSS 1254; Burgasser et al. 2003a) for comparison. The spectra are shown on a log flux scale and have been smoothed to $R \approx 1000$ to match the resolution of 2MASS 1632 observed with Keck I LRIS. Overall, the continuum of 2MASS $1404 \mathrm{AB}$ is intermediate between the L8 and

${ }^{8}$ IRAF is distributed by the National Optical Astronomy Observatory, which is operated by the Association of Universities for Research in Astronomy, Inc., under cooperative agreement with the National Science Foundation. 


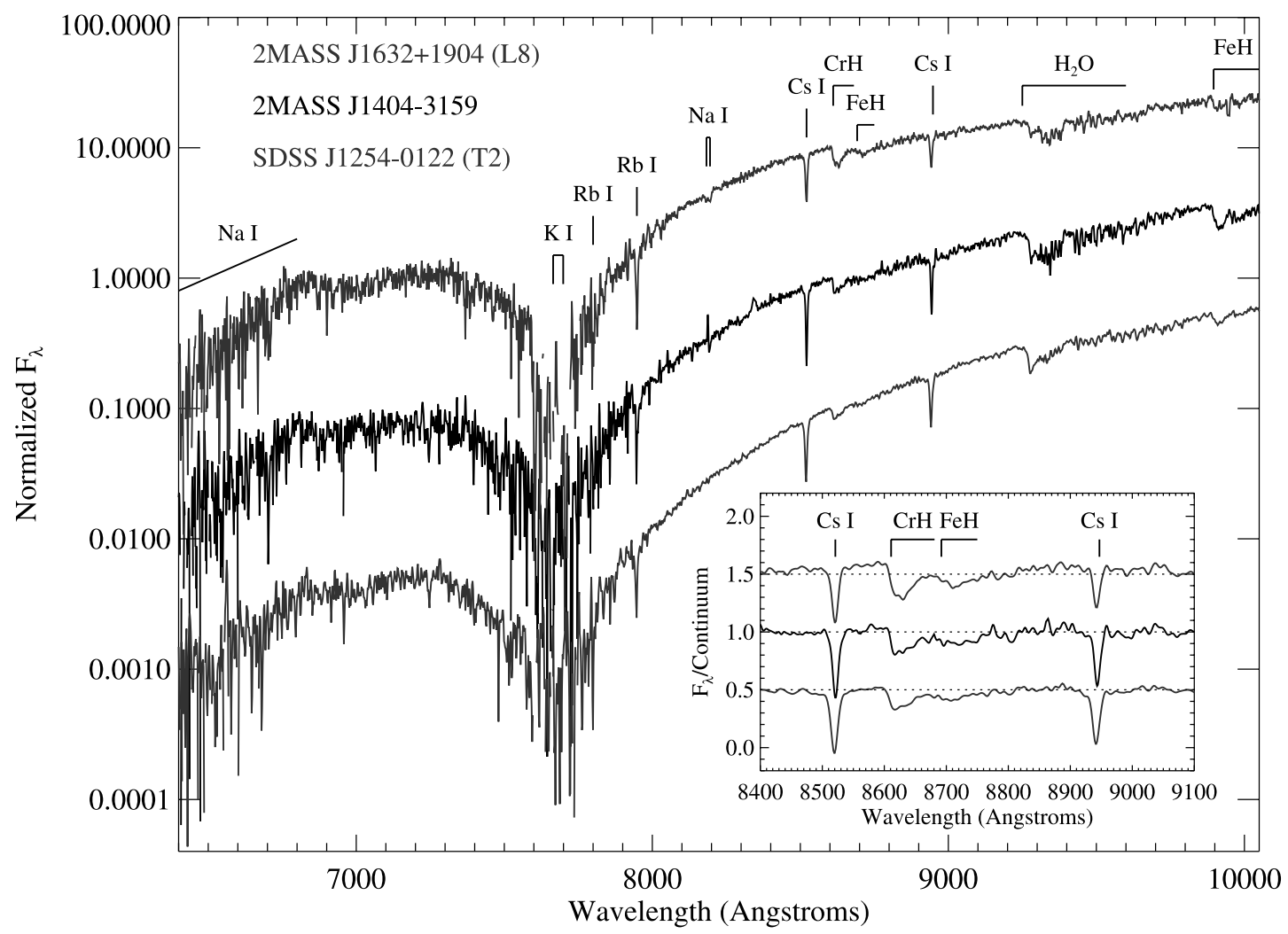

FIG. 3.- Red optical spectrum of 2MASS 1404AB (obtained with LDSS-3/Magellan; optical T0 \pm 1) in comparison to 2MASS 1632 (L8 optical standard; Kirkpatrick et al. 1999) and SDSS 1254 (T2 optical standard; Burgasser et al. 2003a) normalized and shown on a log flux scale. The insert shows the region between 8400 and $9100 \AA$, where the spectra have been divided by a fourth-order polynomial fit to the continua. 2MASS 1404AB and SDSS 1254 have been smoothed down to approximately the same resolution as $2 \mathrm{MASS} 1632(R \approx 1000)$. Major atomic and molecular features are labeled, and the spectra are separated along the vertical axis for clarity. 2MASS $1404 \mathrm{AB}$ appears to be intermediate in type between the L8 and T2 optical standards.

T2 standards, consistent with an optical spectral type of T0. The inset box in this same figure shows the $\mathrm{Cs}$ I lines and $\mathrm{FeH} / \mathrm{CrH}$ bands between 8400 and $9100 \AA$. The spectra shown in this insert have been divided through by a fourth-order polynomial fit to the continuum in order to highlight the strength of these features. The Cs I lines are of comparable strength in 2MASS 1404AB as in SDSS 1254, while the FeH/CrH band in 2MASS 1404AB is intermediate between the L8 and T2 standards. We therefore adopt an optical spectral type of T0 \pm 1 for the 2MASS $1404 \mathrm{AB}$ system.

\section{ANALYSIS}

\subsection{New $M_{J_{H K}}$-Spectral Type Relations Based on 2MASS Photometry}

We have derived $J H K_{s}$ absolute magnitude versus spectral type relations using optically classified late-type $\mathrm{M}$ and $\mathrm{L}$ dwarfs and NIR classified T dwarfs with parallax measurements of signalto-noise ratio $(\mathrm{S} / \mathrm{N})>5$ and not known to be binary (collected from Perryman et al. 1997; Dahn et al. 2002; Tinney et al. 2003; Vrba et al. 2004). ${ }^{9}$ The coefficients of the sixth-degree polynomial fits to this unweighted data are given in Table 3 and shown graphically in Figure 4. We have used optical spectral classification for the L dwarfs because no formal L dwarf NIR spectral classification scheme has been constructed. ${ }^{10}$ These relations use the new NIR spectral classification scheme for T dwarfs (Burgasser et al. 2006b), superseding previous $M_{J H K}$ versus spectral type (SpT)

\footnotetext{
9 See also: Vizier Online Data Catalog, 1174 (W. F. van Altena, J. T. Lee, \& D. Hoffleit, 1995).

${ }^{10}$ Geballe et al. (2002) have identified classification indices based on NIR molecular absorption bands.
}

TABLE 3

Coefficients of Polynomial Fits for L and T Dwarfs

\begin{tabular}{|c|c|c|c|c|c|c|c|c|}
\hline$M_{J}^{\mathrm{a}}(\mathrm{mag}) \ldots \ldots$ & 11.817 & $1.255 \mathrm{E}-1$ & $3.690 \mathrm{E}-2$ & $1.663 \mathrm{E}-2$ & $-3.915 \mathrm{E}-3$ & $2.595 \mathrm{E}-4$ & $-5.462 \mathrm{E}-6$ & 0.29 \\
\hline$M_{H}^{\mathrm{a}}(\mathrm{mag}) \ldots \ldots \ldots$ & 11.010 & $1.125 \mathrm{E}-1$ & $3.032 \mathrm{E}-2$ & $1.261 \mathrm{E}-2$ & $-2.970 \mathrm{E}-3$ & $1.987 \mathrm{E}-4$ & $-4.218 \mathrm{E}-6$ & 0.29 \\
\hline $\mathrm{BC}_{K}^{b, \mathrm{c}}(\mathrm{mag}) \ldots \ldots \ldots \ldots \ldots$ & 3.221 & $2.371 \mathrm{E}-2$ & $6.428 \mathrm{E}-3$ & $-1.631 \mathrm{E}-3$ & $5.579 \mathrm{E}-5$ & $\ldots$ & $\ldots$ & 0.16 \\
\hline 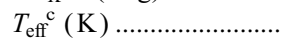 & 2319.920 & -108.094 & 1.950 & -3.101 & $6.414 \mathrm{E}-1$ & $-4.255 \mathrm{E}-2$ & $9.084 \mathrm{E}-4$ & 87 \\
\hline
\end{tabular}

Notes.-Polynomial fits to optical L dwarfs (classified on Kirkpatrick et al. 1999 scheme) and NIR T dwarfs (classified on Burgasser et al. 2006b scheme) with parallax measurements and not known to be binary (see $\S \S 3.1$ and 3.4 for a full description). Each function is defined as $R=\sum_{i=0}^{n} c_{i}(\mathrm{SpT})^{i}$ and is valid for spectral types $\mathrm{L} 0-\mathrm{T} 8$, where $0=\mathrm{L} 0,10=\mathrm{T} 0$, etc. These fits are shown graphically in Figs. 4 and 8.

a Photometry is on the 2MASS system.

${ }^{b}$ Photometry is on the MKO system.

${ }^{\mathrm{c}}$ Data are from Golimowski et al. (2004) and references therein. 

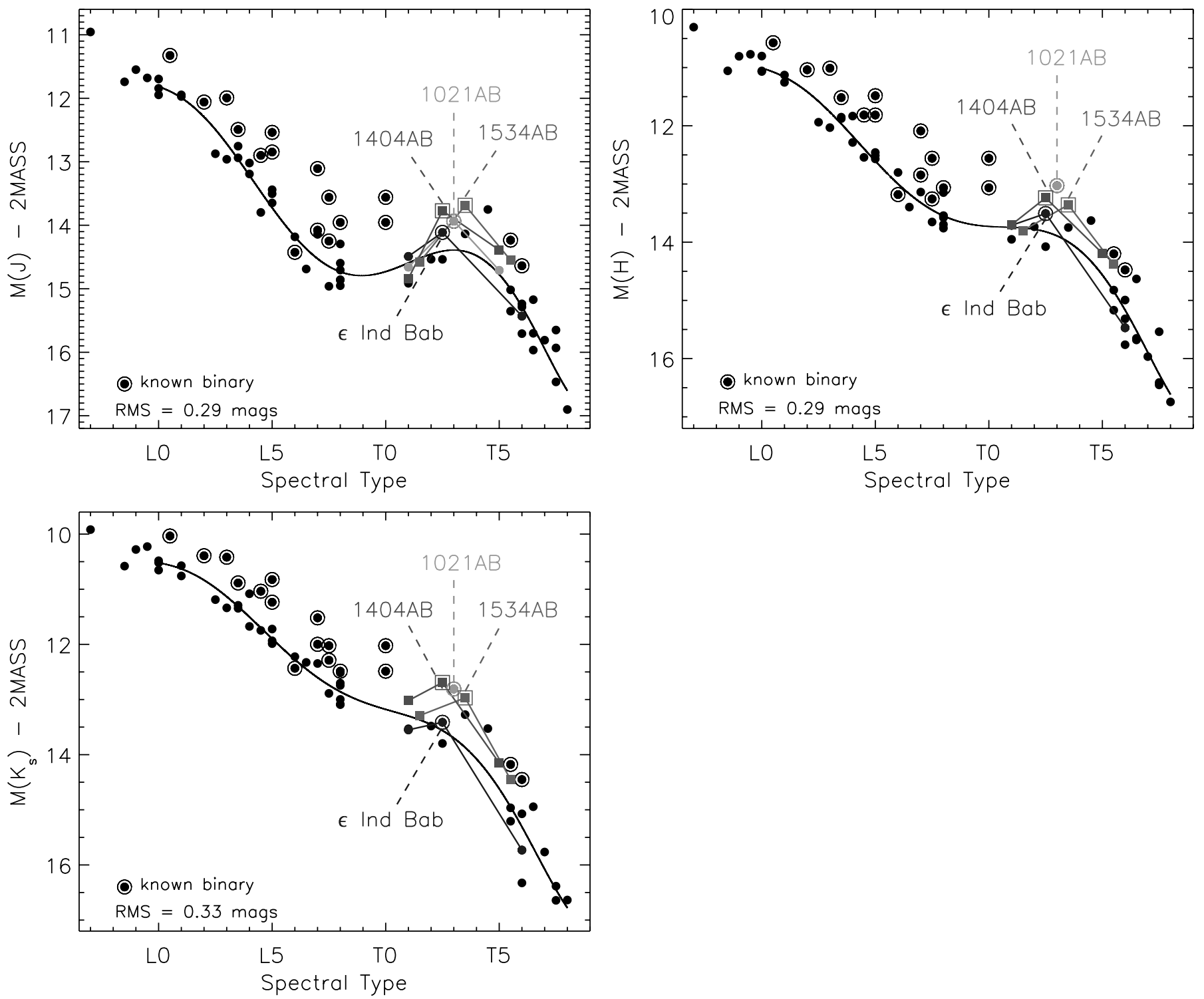

FIG. 4. $-M_{J H K_{s}}$ vs. SpT of optical L dwarfs (on Kirkpatrick et al. 1999 scheme) and NIR T dwarfs (on Burgasser et al. 2006b scheme) with parallax measurements of $\mathrm{S} / \mathrm{N}>5$ ( filled circles). The (unweighted) sixth-degree polynomial fits defined in $\S 3.1$ are shown as solid lines. Late-type M dwarfs were also included in these fits to prevent an artificial downturn at L0. Known binaries are encircled and were not included in the fit, with the exception of $\epsilon$ Ind Bab (black), where the spectroscopically classified components have been used. Top: A clear brightening in $M_{J}$ is seen from $\sim \mathrm{T} 1-\mathrm{T} 5$, known as the $J$-band "bump." The three known $J$-band flux reversal binariesSDSS 1021AB, SDSS 1534AB, and 2MASS 1404AB - and their A+B components are shown as light gray, medium gray, and dark gray, respectively. 2MASS 1404AB and SDSS $1534 \mathrm{AB}$ are indicated by squares, not circles, since no parallax measurement has been made for either of these sources. No $H$-and $K_{s}$-band resolved photometry is available for SDSS 1021AB. [See the electronic edition of the Journal for a color version of this figure.]

relations on the old NIR T dwarf scheme (Geballe et al. 2002; Burgasser et al. 2002).

\subsection{Component Spectral Types of 2MASS 1404AB}

Since the relative $J$ - and $H$-band photometry is on the MKO photometric system and the relative $K$-band photometry is on the
2MASS photometric system, we need to convert the $K$-band photometry onto the same MKO system. To do this, we examined a set of 25 L6.0-T2.0 dwarfs, described below, and computed the (2MASS - MKO) $K$-band color term as $-0.01 \pm 0.04$ averaged over these spectral classes. We show the breakdown of this color term per spectral class in Table 4. Since this color term difference

TABLE 4

$K$-Band Differences between 2MASS and MKO by Spectral Type: L6.0-T2.0

\begin{tabular}{|c|c|c|c|c|c|c|c|c|}
\hline Quantity & L6 & L7 & L8 & L9 & T0 & $\mathrm{T} 1$ & $\mathrm{~T} 2$ & Average \\
\hline 2 MASS $K_{s}-\operatorname{MKO} K \ldots \ldots \ldots \ldots \ldots$ & $0.03 \pm 0.01$ & 0.02 & $0.02 \pm 0.01$ & $0.01 \pm 0.01$ & 0.01 & $-0.01 \pm 0.02$ & $-0.03 \pm 0.02$ & $-0.01 \pm 0.04$ \\
\hline Number of objects ${ }^{\mathrm{a}} \ldots \ldots \ldots \ldots \ldots \ldots$ & 4 & 1 & 2 & 2 & 1 & 7 & 8 & 25 \\
\hline
\end{tabular}

a The number of objects available per spectral type class, where a class also includes a half-subtype-i.e., L6.0 and L6.5. Note that the T 2 spectral class only includes T2.0 objects and no T2.5 objects. 
TABLE 5

Properties of the Components of the 2MASS 1404AB System

\begin{tabular}{|c|c|c|}
\hline Property & 2MASS $1404 \mathrm{~A}$ & 2MASS 1404B \\
\hline MKO $J(\mathrm{mag}) \ldots \ldots .$. & $16.46 \pm 0.12$ & $15.93 \pm 0.09$ \\
\hline MKO $H$ (mag) ................................. & $15.56 \pm 0.09$ & $16.04 \pm 0.10$ \\
\hline 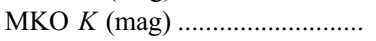 & $14.86 \pm 0.11$ & $16.06 \pm 0.19$ \\
\hline 2MASS $J$ (mag) ........... & $16.65 \pm 0.12$ & $16.20 \pm 0.09$ \\
\hline 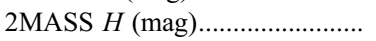 & $15.51 \pm 0.09$ & $16.00 \pm 0.10$ \\
\hline 2 MASS $K_{s}(\mathrm{mag}) \ldots \ldots \ldots \ldots \ldots \ldots \ldots$ & $14.83 \pm 0.11$ & $15.96 \pm 0.19$ \\
\hline 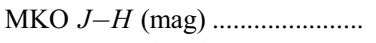 & $0.90 \pm 0.15$ & $-0.11 \pm 0.13$ \\
\hline MKO $H-K(\mathrm{mag})$ & $0.70 \pm 0.14$ & $-0.02 \pm 0.21$ \\
\hline MKO $J-K(\mathrm{mag}) \ldots \ldots \ldots \ldots \ldots \ldots \ldots$ & $1.60 \pm 0.16$ & $-0.13 \pm 0.21$ \\
\hline 2MASS $J-H$ (mag) ....................... & $1.14 \pm 0.15$ & $0.20 \pm 0.13$ \\
\hline 2MASS $H-K_{S}(\mathrm{mag}) \ldots \ldots \ldots \ldots \ldots$ & $0.68 \pm 0.14$ & $0.04 \pm 0.21$ \\
\hline 2 MASS $J-K_{S}(\mathrm{mag}) \ldots \ldots \ldots \ldots \ldots \ldots$ & $1.82 \pm 0.16$ & $0.24 \pm 0.21$ \\
\hline Est. NIR SpT ${ }^{\mathrm{a}} \ldots \ldots \ldots$ & $\mathrm{T} 1 \pm 1$ & $\mathrm{~T} 5 \pm 1$ \\
\hline Est. $T_{\text {eff }}(\mathrm{K})^{\mathrm{b}} \ldots \ldots$ & $1390 \pm 90$ & $1180 \pm 90$ \\
\hline$M_{\mathrm{bol}}(\mathrm{mag})$ & $15.96 \pm 0.19$ & $16.59 \pm 0.25$ \\
\hline
\end{tabular}

${ }^{a}$ Component NIR spectral types are derived in $\S 3.2$.

b Based on the $T_{\text {eff }}$ vs. SpT relation defined in Table 3, using the component spectral types listed and assuming an age of $3 \mathrm{Gyr}$.

is near zero, we assume the two are equivalent and proceed to determining the component $\mathrm{MKO}$ photometry, using the following steps:

1. Convert $J_{\mathrm{AB}}(2 \mathrm{MASS})$ to $J_{\mathrm{AB}}(\mathrm{MKO})$ using the (MKO 2MASS) color term computed from the spectrum of 2MASS 1404AB (Looper et al. 2007): $J_{\mathrm{AB}}(\mathrm{MKO}-2 \mathrm{MASS})=-0.17$; $J_{\mathrm{AB}}(\mathrm{MKO})=15.41 \pm 0.07$.

2. Decompose the system: $J_{\mathrm{A}}=J_{\mathrm{AB}}+2.5 \log \left(1+10^{0.4 \times \Delta J}\right)$; $\sigma_{J_{\mathrm{A}}}^{2}=\sigma_{J_{\mathrm{AB}}}^{2}+\left[\sigma_{\Delta J}^{2} /\left(10^{-0.4 \times \Delta J}-1\right)^{2}\right]$, where $\Delta J=J_{\mathrm{A}}-J_{\mathrm{B}}$; $J_{\mathrm{A}}(\mathrm{MKO})=16.46 \pm 0.12$.

3. Similarly, $J_{\mathrm{B}}=J_{\mathrm{AB}}+2.5 \log \left(1+10^{-0.4 \times \Delta J}\right) ; \sigma_{J_{\mathrm{B}}}^{2}=\sigma_{J_{\mathrm{AB}}}^{2}+$ $\left[\sigma_{\Delta J}^{2} /\left(10^{0.4 \times \Delta J}-1\right)^{2}\right]$, where $\Delta J=J_{\mathrm{A}}-J_{\mathrm{B}} ; J_{\mathrm{B}}(\mathrm{MKO})=$ $15.93 \pm 0.09$.

In the same manner, this procedure was carried out to determine the MKO $H$-band and $K$-band component photometry (see Table 5).

To estimate NIR spectral types for the AB components, we compared the integrated light spectrum to a suite of synthetic Infrared Telescope Facility SpeX spectra constructed in the same manner as Burgasser et al. (2008). The constituents for these synthetic spectra are the 25 L6-T2 dwarfs, used for the primaries, and the $38 \mathrm{~T} 3-\mathrm{T} 8$ dwarfs, used for the secondaries, as listed in Burgasser et al. (2008), ${ }^{11}$ which is drawn from Burgasser et al. (2004, 2006a, 2006b, 2007), Chiu et al. (2006), Cruz et al. (2004), Liebert \& Burgasser (2007), Looper et al. (2007), Reid et al. (2006), and Siegler et al. (2007). In cases where more than one spectrum for an object was available, we used the highest $\mathrm{S} / \mathrm{N}$ spectrum available. All combinations of primary and secondary were scaled together using the $M_{J}$ magnitude from the $M_{J}$-SpT relation derived above in $\S 3.1$. This resulted in 1000 synthetic spectra. To estimate the goodness of fit between the data (2MASS 1404AB) and each model, we first interpolated the flux of the models onto the wavelength scale of the data and normalized each to their peak flux over the $1.2-1.3 \mu \mathrm{m}$ window. We then calculated the $\chi^{2}$

\footnotetext{
11 See also http://web.mit.edu/ajb/www/browndwarfs/spexprism/htm/ binarytemplates.html for a list of these 63 spectra, where we have eliminated 2MASS J21513839-4853542 (T4) and 2MASS J05103520-4208140 ( T5 ) from the list of T3-T8 dwarfs used due to the poor $\mathrm{S} / \mathrm{N}$ of their spectra.
}

values ${ }^{12}$ between the data and each model over the wavelength ranges $0.95-1.35,1.45-1.8$, and $2.0-2.35 \mu \mathrm{m}$ to avoid low $\mathrm{S} / \mathrm{N}$ regions of the spectra. In addition, we calculated $\chi^{2}$ for a range of multiplicative scale factors $(0.5-1.5)$ of the data, in steps of 0.01 , to eliminate the normalization bias, selecting the normalization that yielded $\chi_{\min }^{2}$.

From the 1000 model spectra, we selected the 15 spectra with the smallest $\chi^{2}$ values for visual inspection. The model spectrum created from SDSS J205235.31-160929.8 ( T1, hereafter SDSS 2052; Chiu et al. 2006) for the primary and from 2MASS J23312378-4718274 (T5, hereafter 2MASS 2331; Burgasser et al. 2004) for the secondary with the same normalization provided the best fit and yielded 2MASS $\Delta J=0.18$ mag. We also examined model combinations constructed from two earlier type (L9 and T0) and two later type (T1.5 and T2) primaries with a T5 used as the secondary. In addition, we examined model combinations constructed from a T1 as the primary and two earlier type (T4 and T4.5) and two later type (T5.5 and T6) secondaries. Since many spectra with identical spectral types were available, we used those with model spectra that minimized $\chi^{2}$. All fits are shown in Figure 5. The best fit is clearly the T1/T5 model, which shows an excellent match to the data of $2 \mathrm{MASS} 1404 \mathrm{AB}$ over all wavelengths. Deviating the spectral type of either the primary or secondary by a subtype or more while holding the other fixed, results in a degraded goodness of fit to the data. We therefore estimate a final NIR spectral type of the primary as $\mathrm{T} 1 \pm 1$ and of the secondary as $\mathrm{T} 5 \pm 1$.

Using the MKO component photometry of 2MASS 1404AB and component spectral type estimates of T1 and T5, we compute the 2MASS component photometry (see Table 5) using the appropriate transformations from Stephens \& Leggett (2004). These relations are dependent on spectral type with errors that add negligibly to the component photometric errors. We can compare the relative 2MASS $J$-band photometry obtained from imaging, $\Delta J=0.45 \pm 0.15$, to that obtained from our synthetic spectral modeling, $\Delta J=0.18$, using a $\mathrm{T} 1$ primary and a T5 secondary. The relative $2 \mathrm{MASS} J$-band photometry obtained from our synthetic spectral modeling is $1.8 \sigma$ from our relative 2MASS $J$-band photometry obtained from imaging. It confirms that the secondary is indeed brighter than the primary in $J$ band.

As a check on our spectral type estimates, we compute 2MASS NIR colors of each component and compare these to the 2MASS NIR colors of published L and T dwarfs ${ }^{13}$ in Figure 6. In this colorcolor diagram, component $\mathrm{A}$ is seen coincident with a cluster of L7-T1 dwarfs. Component B is associated with a cluster of T4T6 dwarfs. This color comparison yields an average spectral type estimate of $\sim \mathrm{L} 9$ for component $\mathrm{A}$ and $\sim \mathrm{T} 5$ for component $\mathrm{B}$.

In Figure 7 we plot SDSS 2052 and 2MASS 2331 scaled with the relative photometry of the A and B components of 2MASS $1404 \mathrm{AB}$, with the 2MASS and MKO filter transmission profiles overlaid. The $2 \mathrm{MASS} J$-band filter profile extends to slightly shorter wavelengths, covering more of the $\mathrm{CH}_{4}$ absorption band at $\sim 1.15 \mu \mathrm{m}$, than does the MKO $J$-band profile. This explains the reduced relative 2 MASS $J$-band photometry of the two components compared to the relative MKO $J$-band photometry, since the secondary ( $\mathrm{T} 5$ ) has larger $\mathrm{CH}_{4}$ absorption than does the primary (T1). The flux peaks, however, are more illuminating than

\footnotetext{
12 Defined as $\chi^{2} \equiv \Sigma_{\lambda}\left\{\left[f_{\lambda}(1404 \mathrm{AB})-f_{\lambda}(M)\right] / \sigma_{\lambda}\right\}^{2}$, where $f_{\lambda}(1404 \mathrm{AB})$ is the data for 2MASS 1404AB, $f_{\lambda}(M)$ is the synthetic model spectrum, and $\sigma_{\lambda}$ are the errors in the data for 2MASS 1404AB. The summation is performed over the wavelengths $0.95-1.35,1.45-1.8$, and $2.0-2.35 \mu \mathrm{m}$.

13 See http://dwarfarchives.org.
} 


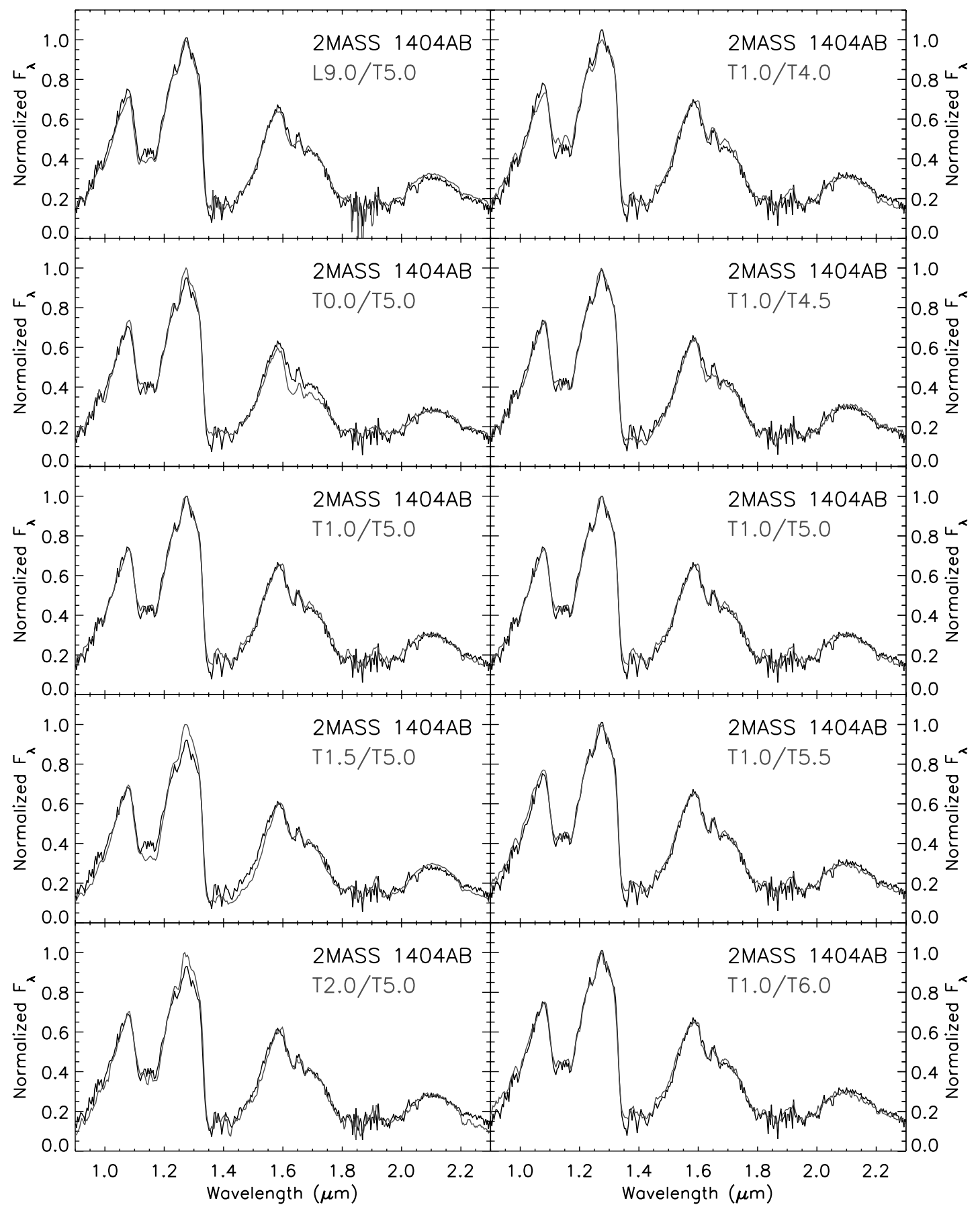

FIG. 5.-Comparison of the T2.5 integrated light spectrum of 2MASS 1404AB (black; Looper et al. 2007) with the best synthetic spectral fits ( gray; see § 3.2). The T1 and T5 combination provides the best fit across all wavelengths to the spectrum of 2MASS 1404AB. [See the electronic edition of the Journal for a color version of this figure.]

the broadband photometry. The peak at $1.25 \mu \mathrm{m}$ of the scaled T5 is $\sim 70 \%$ brighter than that of the scaled $\mathrm{T} 1$, and the $1.05 \mu \mathrm{m}$ peak (outside the $J$-band filter profile) is $\sim 40 \%$ brighter. This redistribution of flux into the 1.05 and $1.25 \mu \mathrm{m}$ peaks is remarkably similar to that seen for SDSS $1021 \mathrm{AB}$ and SDSS 1534AB. SDSS $1021 \mathrm{~B}$ is $\sim 30 \%$ and $\sim 25 \%$ brighter at the 1.25 and $1.05 \mu \mathrm{m}$ bands than SDSS 1021A, respectively. Likewise, SDSS 1534B is 30\% and $\sim 20 \%$ brighter at the 1.25 and $1.05 \mu \mathrm{m}$ bands than SDSS 1534A. While no atmospheric models can accurately reproduce this flux redistribution, we refer the reader to Burgasser et al. (2006c) for a qualitative description of how this brightening may arise.

\subsection{Construction of Color-Magnitude Diagrams}

Using the new $M_{J K_{s}}$-SpT relations derived in $\S 3.1$, we estimate distances for component $\mathrm{A}$, assuming a $\mathrm{T} 1$ spectral type, of $\sim 25.8, \sim 22.6$, and $\sim 20.2 \mathrm{pc}$, respectively, yielding a mean distance of $\sim 23 \mathrm{pc}$. We can now place 2MASS $1404 \mathrm{AB}$ and its components on a color-magnitude diagram (see Fig. 4). Likewise, SDSS $1021 \mathrm{AB}$ and SDSS $1534 \mathrm{AB}$ are also shown, and these have been converted onto the 2MASS photometric system using the spectral type estimates of their components, their component MKO photometry, and the appropriate transformations from Stephens \& Leggett (2004). The parallax measurement for SDSS $1021 \mathrm{AB}$ 


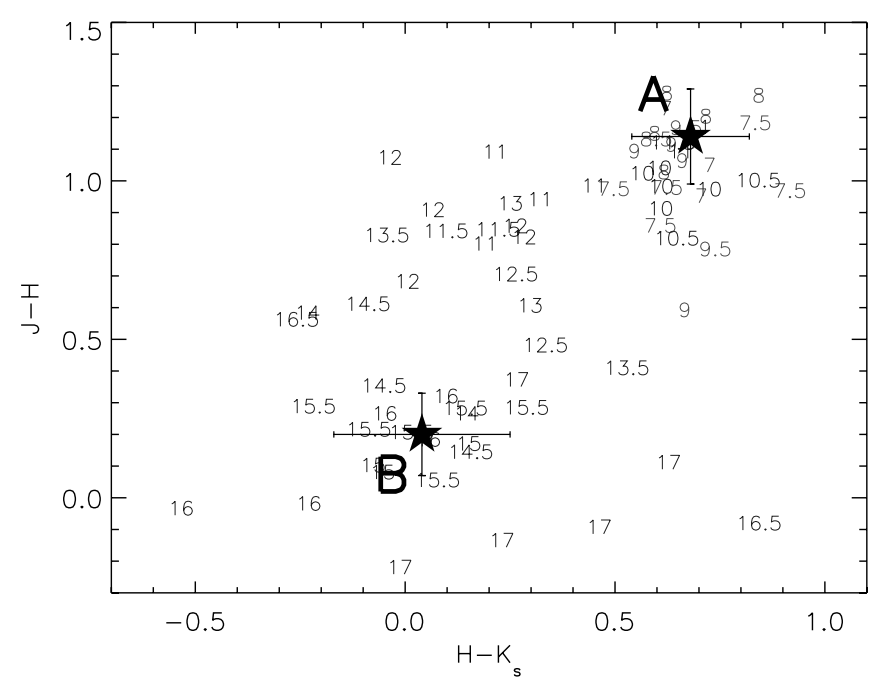

FIG. 6.-NIR color-color plot of 74 known NIR L7-T 7 dwarfs with $\sigma_{J-H}$ and $\sigma_{H-K_{s}}<0.3 \mathrm{mag}$ and spectral types with uncertainties $\leq 1$ subtype. $\mathrm{L}$ dwarfs are shown in light gray with spectral types represented by numbers $-7=\mathrm{L} 7,8=\mathrm{L} 8$, etc. T dwarfs are shown in dark gray with spectral types represented by numbers$10=\mathrm{T} 0,15=\mathrm{T} 5$, etc. The resolved colors of 2MASS 1404AB are shown as stars with associated error bars. [See the electronic edition of the Journal for a color version of this figure.]

(Tinney et al. 2003) is used to place it on the diagram. Since SDSS 1534AB lacks a parallax measurement, we use the $M_{J H K_{s}}$-SpT relations for component SDSS $1534 \mathrm{~A}\left(d_{\mathrm{est}} \approx 41 \mathrm{pc}\right)$ to place SDSS $1534 \mathrm{AB}$ on the diagram.

Of note, only two other T dwarfs in the $J$-band bump, SDSS J175032.96+175903.9 (T3.5, hereafter SDSS 1750) and 2MASS J05591914-1404488 (T4.5, hereafter 2MASS 0559), lie above the $J$-band fit, by $\sim 0.3$ and $\sim 0.9 \mathrm{mag}$, respectively. Neither object has been resolved in high angular resolution studies (Burgasser et al. 2003b, 2006c), nor show any signs of unusual metallicity or gravity effects in their spectra. These objects could still be binaries with very small separations and/or imaged at an unpropitious place in their orbits, as was originally the case with Kelu-1 (Liu \& Leggett 2005; Gelino et al. 2006). However, Zapatero Osorio et al. (2007) have monitored 2MASS 0559 over a period of $4.37 \mathrm{yr}$ with radial velocity measurements and have ruled out companions more massive than $10 M_{\text {Jup }}$ in orbits of $\sim 1 \mathrm{yr}$ and companions more massive than $2 M_{\text {Jup }}$ in orbits of less than a few days.

In order to eliminate the $J$-band bump, SDSS 1750 would have to be an unresolved binary, 2MASS 0559 would have to be an unresolved triple (since $\Delta J>0.75 \mathrm{mag}$ ) with the above restrictions on the orbital periods of its components, and 2MASS 1404AB would also have to be a triple system instead of a binary. The small observed frequency of brown dwarf triple systems $\left(3_{-1}^{+4} \%-\right.$ not considering selection effects; Burgasser et al. 2007) suggests this is highly unlikely. Hence, the amplitude of the $J$-band bump is probably at least $\sim 0.5 \mathrm{mag}$, as illustrated by the components of 2MASS 1404AB and likely as high as $\sim 1$ mag in light of the recent null result of radial velocity companions to 2MASS 0559 by Zapatero Osorio et al. (2007). A parallax measurement and resolved spectroscopy of this system are needed to accurately place the components on a color-magnitude diagram. Radial velocity monitoring of the 2MASS 1404AB system can resolve if this brightening is intrinsic to the atmospheres as they cool, or if higher order multiplicity is responsible for this peculiar flux reversal at $1-1.2 \mu \mathrm{m}$.

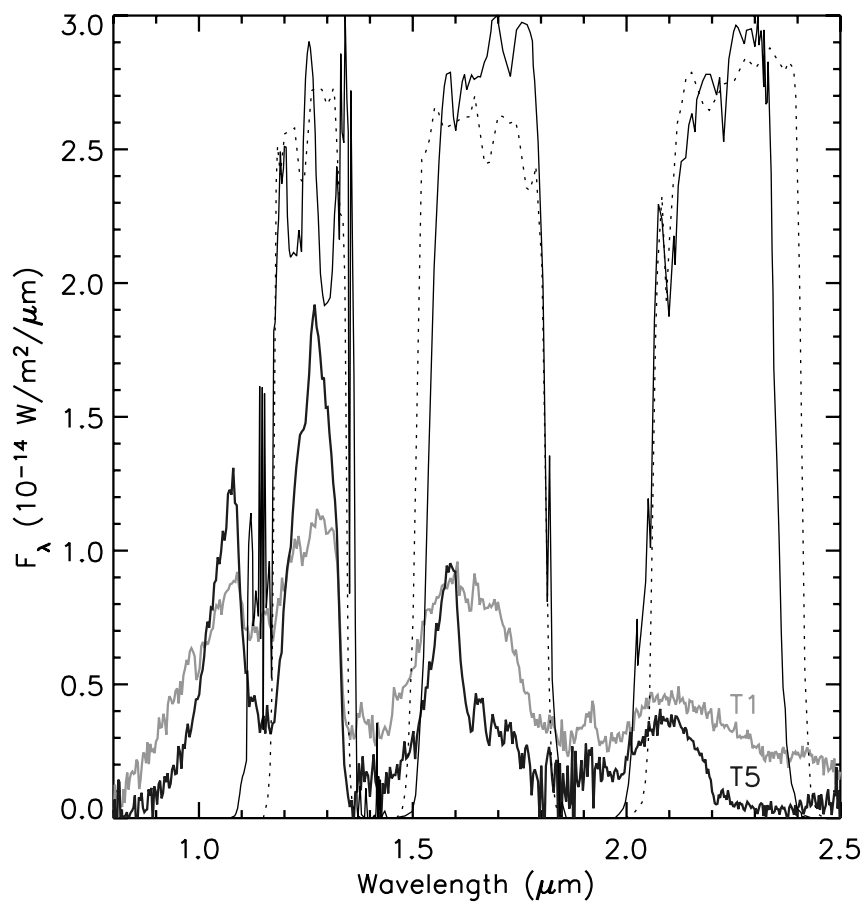

FIG. 7.-SDSS J205235.31-160929.8 (NIR T1; Chiu et al. 2006), shown in light gray, and 2MASS J23312378-4718274 (NIR T5; Burgasser et al. 2004), shown in dark gray, scaled by the MKO magnitudes of the A and B components of 2MASS 1404AB. The 2MASS JHK (solid lines) and MKO JHK (dotted lines) transmission plus atmospheric profiles are overlaid. [See the electronic edition of the Journal for a color version of this figure.]

\subsection{Physical Parameters of 2 MASS $1404 A B$}

To estimate the physical parameters of 2MASS 1404AB (see Table 2), we first rederived $\mathrm{MKO} K$-band bolometric corrections $\left(\mathrm{BC}_{K}\right)$ versus spectral type and $T_{\text {eff }}$ versus spectral type relations, using M9-T8 data from Golimowski et al. (2004) and references therein, excluding known binaries. In both relations, we classify L dwarfs on the Kirkpatrick et al. (1999) optical classification scheme, and T dwarfs on the Burgasser et al. (2006b) NIR classification scheme. Our choice of optical L dwarf spectral classification for these fits is further strengthened because the far-optical spectrum of mid- to late-type L dwarfs is less influenced by cloud opacities than the Geballe et al. (2002) $1.5 \mu \mathrm{m} \mathrm{H}_{2} \mathrm{O}$ index (Knapp et al. 2004). The coefficients to the fourth-degree weighted $\mathrm{BC}_{K}$ polynomial fit and to the sixth-degree unweighted $T_{\text {eff }}$ polynomial fit are given in Table 3 and shown graphically in Figure 8. The rms in this fit ( $87 \mathrm{~K}$ ) compared to that of Golimowski et al. (2004; $124 \mathrm{~K}$ ) is less because of the elimination of objects found to be binary between the time of that publication and this paper. Using the $T_{\text {eff }}-\mathrm{SpT}$ relation, we estimate effective temperatures of $\sim 1390 \pm 90$ and $\sim 1180 \pm 90 \mathrm{~K}$ for the primary and secondary, respectively, assuming an age of $3 \mathrm{Gyr}$. We also find $\mathrm{BC}_{K}$ values of $2.91 \pm 0.16$ and $2.34 \pm 0.16$ for the primary and secondary, respectively. In both cases, the error is the rms in the fit and does not include the error in spectral typing.

To determine $M_{\text {bol }}$ for each component, we used the $\mathrm{BC}_{K}$ corrections, a distance estimate of $d \approx 23 \mathrm{pc}$, and the MKO component photometry (see Table 5). This yielded $M_{\mathrm{bol}}=15.96 \pm$ 0.19 and $16.59 \pm 0.25 \mathrm{mag}$ for the primary and secondary, respectively. The ratio of bolometric luminosities, $L_{\mathrm{bol}, \mathrm{A}} / L_{\mathrm{bol}, \mathrm{B}}$, is $1.79 \pm 0.52$, with the primary having the higher luminosity. We use the mass-luminosity relation $L_{\text {bol }} \propto M^{2.64}$ (Burrows et al. 


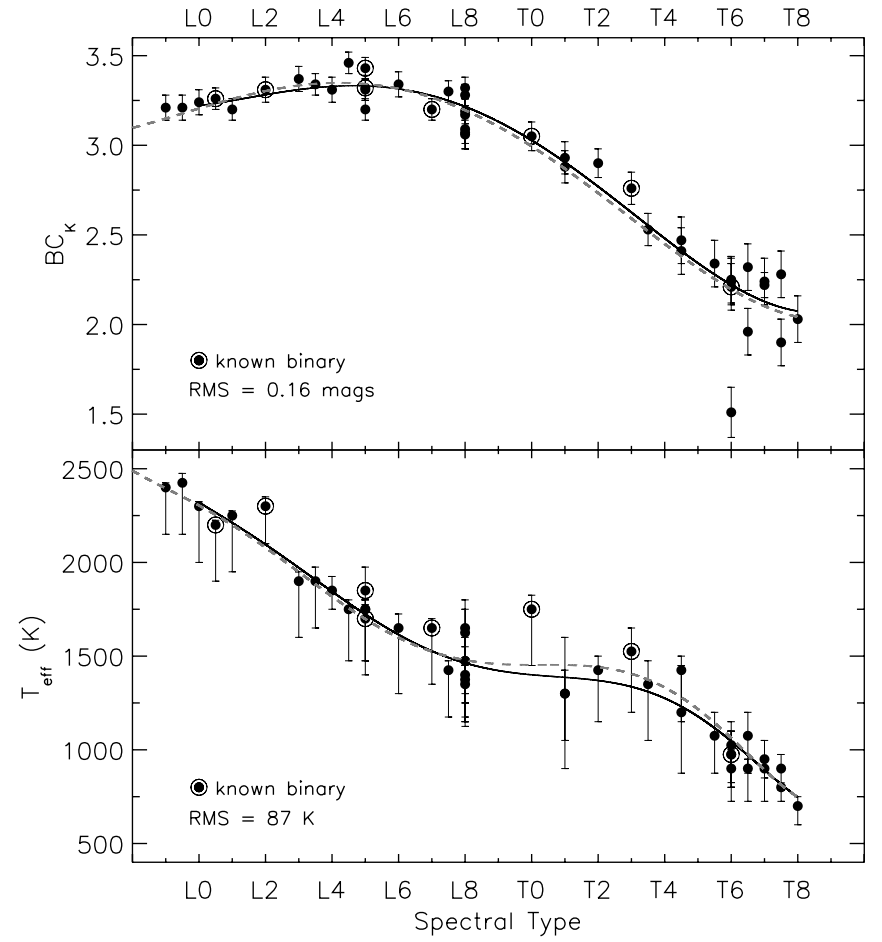

Fig. 8.-Polynomial fits (solid lines) to $\mathrm{M} 9-\mathrm{T} 8$ objects with $\mathrm{BC}_{K}$ measurements and $T_{\text {eff }}$ estimates (from Golimowski et al. 2004 and references therein), excluding known binaries. The relations are fourth- and sixth-degree polynomial fits for $\mathrm{BC}_{K}$ and $T_{\text {eff }}$, respectively, with the coefficients listed in Table 3. The plotted values used in the $T_{\text {eff }}$ relation are for an age of $3 \mathrm{Gyr}$, and the error bars represent an age range of 0.1-10 Gyr (younger ages are cooler, and older ages are hotter). The $\mathrm{BC}_{K}$-SpT relation is weighted by the errors, while the $T_{\text {eff }}-\mathrm{SpT}$ relation is unweighted. The large outlier seen in the $\mathrm{BC}_{K}$ plot and included in the fit is the T6 dwarf 2MASSI J0937347+293142, which is thought to be unusually blue due to high surface gravity/low metallicity. Shown for comparison are the polynomial fits (dashed lines) from Golimowski et al. (2004).

2001), which assumes solar metallicities, and the ratio of bolometric luminosities to find the mass ratio: $q \equiv M_{\mathrm{B}} / M_{\mathrm{A}}=0.80 \pm$ 0.09 . This mass ratio is typical for known brown dwarf binaries, which tend to peak at $q \sim 1$ ( $73 \%$ of observed binaries have $q \geq 0.8$, although observational bias would favor high- $q$ ratios; Burgasser et al. 2006c). Using the age-luminosity relation from Burrows et al. (2001) we find a total mass for the system of 50, 70 , and $80 M_{\text {Jup }}$, corresponding to ages of $0.5,1.0$, and $5.0 \mathrm{Gyr}$, typical for local disk dwarfs (Reid \& Hawley 2000). This corresponds to individual masses of $\sim 28$ and $22 M_{\text {Jup }}, \sim 39$ and $31 M_{\text {Jup }}$, and $\sim 44$ and $36 M_{\text {Jup }}$ for these respective ages.

Finally, this allows determination of the orbital parameters of this system. At an estimated distance of $\sim 23 \mathrm{pc}$ and with an apparent separation of $0.134^{\prime \prime}$, the projected physical separation is $\rho \approx 3.1$ AU. Statistically, the true semimajor axis can then be estimated as $a=1.26 \times \rho \approx 3.9 \mathrm{AU}$ (Fischer \& Marcy 1992). This implies an orbital period of roughly $28-35 \mathrm{yr}$, assuming a total system mass of 50-80 $M_{\text {Jup }}$.

\section{CONCLUSIONS}

We have presented high-resolution imaging of two T2.5 dwarfs-2MASS 1106 and 2MASS 1404-and resolved the latter into a $0.13^{\prime \prime}$ binary. $2 \mathrm{MASS} 1404 \mathrm{AB}$ is an intriguing binary, as its presumably cooler secondary is brighter in $J$ band than the primary by $2 \mathrm{MASS} \Delta J=0.45 \mathrm{mag}$, but fainter in both 2MASS $H$ (by $0.49 \mathrm{mag}$ ) and 2MASS $K_{s}$ bands (by $1.13 \mathrm{mag}$ ). This secondary brightening in $J$ band is much more pronounced than that seen in the other two known flux reversal binaries with resolved $J$-band photometry - SDSS 1021 (2MASS $\Delta J=-0.05$, MKO $\Delta J=0.04$ ) and SDSS 1534 (2MASS $\Delta J=0.03$, MKO $\Delta J=0.17$ ). Zapatero Osorio et al. (2007) have found no companions in their radial velocity monitoring of the T4.5 dwarf 2MASS 0559, which lies $\sim 0.9$ mag above the 2MASS $M_{J}$ versus spectral type relation we derive. This result in conjunction with ours suggests that a brightening of at least $\sim 0.5$ mag and likely as high as $\sim 1 \mathrm{mag}$ in the $J$-band bump is real and intrinsic to the atmospheres of these objects as they cool across early- to mid-type $\mathrm{T}$ classes.

We wish to thank our anonymous referee for helpful suggestions that have improved the quality of this paper. We would like to thank our telescope operators on Keck: Jim Lyke, Cynthia Wilburn, Chuck Sorenson, and the rest of the Keck LGS AO team, and our telescope operator on Magellan: Mauricio Martinez. We thank Michael Liu for the LGS AO data on these two sources, and Michael Cushing and Mark Pitts for helpful discussions. D. L. L. thanks Michael Liu and John Rayner for advising her for this project, and David Sanders for financial support. This research has made use of the SIMBAD database, operated at CDS, Strasbourg, France. This research has benefitted from the $\mathrm{M}$, L, and T dwarf compendium housed at DwarfArchives.org, and has used data products from the SpeX Prism Spectral Libraries (http://www.browndwarfs.org/spexprism). This publication also makes use of data products from the Two Micron All Sky Survey (2MASS), which is a joint project of the University of Massachusetts and the Infrared Processing and Analysis Center/ California Institute of Technology, funded by the National Aeronautics and Space Administration and the National Science Foundation. This research has also made use of the NASA/ IPAC Infrared Science Archive (IRSA), which is operated by the Jet Propulsion Laboratory, California Institute of Technology, under contract with the National Aeronautics and Space Administration. As all data were obtained from the summit of Mauna Kea, the authors wish to recognize and acknowledge the very significant cultural role and reverence that this mountaintop has always had within the indigenous Hawaiian community. We are most fortunate to have the opportunity to conduct observations on the summit.

Facilities: Keck:II, Magellan:Clay
Ackerman, A. S., \& Marley, M. S. 2001, ApJ, 556, 872

Allington-Smith, J., et al. 1994, PASP, 106, 983

Burgasser, A. J. 2007, ApJ, 659, 655

Burgasser, A. J., Burrows, A., \& Kirkpatrick, J. D. 2006a, ApJ, 639, 1095

Burgasser, A. J., Geballe, T. R., Leggett, S. K., Kirkpatrick, J. D., \& Golimowski, D. A. 2006b, ApJ, 637, 1067

Burgasser, A. J., Kirkpatrick, J. D., Cruz, K. L., Reid, I. N., Leggett, S. K., Liebert, J., Burrows, A., \& Brown, M. E. 2006c, ApJS, 166, 585

\section{REFERENCES}

Burgasser, A. J., Kirkpatrick, J. D., Liebert, J., \& Burrows, A. 2003a, ApJ, 594, 510

Burgasser, A. J., Kirkpatrick, J. D., Reid, I. N., Brown, M. E., Miskey, C. L., \& Gizis, J. E. 2003b, ApJ, 586, 512

Burgasser, A. J., Liu, M. C., Ireland, M. J., Cruz, K. L., \& Dupuy, T. J. 2008, ApJ, 681, 579

Burgasser, A. J., Marley, M. S., Ackerman, A. S., Saumon, D., Lodders, K., Dahn, C. C., Harris, H. C., \& Kirkpatrick, J. D. 2002, ApJ, 571, L151 
Burgasser, A. J., McElwain, M. W., Kirkpatrick, J. D., Cruz, K. L., Tinney, C. G., \& Reid, I. N. 2004, AJ, 127, 2856

Burgasser, A. J., Reid, I. N., Siegler, N., Close, L., Allen, P., Lowrance, P., \& Gizis, J. 2007, in Protostars and Planets V, ed. B. Reipurth, D. Jewitt, \& K. Keil (Tucson: Univ. Arizona Press), 427

Burrows, A., Hubbard, W. B., Lunine, J. I., \& Liebert, J. 2001, Rev. Mod. Phys., 73, 719

Burrows, A., Sudarsky, D., \& Hubeny, I. 2006, ApJ, 640, 1063

Chiu, K., Fan, X., Leggett, S. K., Golimowski, D. A., Zheng, W., Geballe, T. R., Schneider, D. P., \& Brinkmann, J. 2006, AJ, 131, 2722

Cruz, K. L., Burgasser, A. J., Reid, I. N., \& Liebert, J. 2004, ApJ, 604, L61

Dahn, C. C., et al. 2002, AJ, 124, 1170

Fischer, D. A., \& Marcy, G. W. 1992, ApJ, 396, 178

Geballe, T. R., et al. 2002, ApJ, 564, 466

Gelino, C. R., Kulkarni, S. R., \& Stephens, D. C. 2006, PASP, 118, 611

Gizis, J. E., Reid, I. N., Knapp, G. R., Liebert, J., Kirkpatrick, J. D., Koerner, D. W., \& Burgasser, A. J. 2003, AJ, 125, 3302

Golimowski, D. A., et al. 2004, AJ, 128, 1733

Hamuy, M., Suntzeff, N. B., Heathcote, S. R., Walker, A. R., Gigoux, P., \& Phillips, M. M. 1994, PASP, 106, 566

Kirkpatrick, J. D. 2005, ARA\&A, 43, 195

Kirkpatrick, J. D., et al. 1999, ApJ, 519, 802
Knapp, G. R., et al. 2004, AJ, 127, 3553

Liebert, J., \& Burgasser, A. J. 2007, ApJ, 655, 522

Liu, M. C., \& Leggett, S. K. 2005, ApJ, 634, 616

Liu, M. L., Leggett, S. K., Golimowski, D. A., Chiu, K., Fan, X., Gellae, T. R., Schneider, D. P., \& Brinkmann, J. 2006, ApJ, 647, 1393

Looper, D. L., Kirkpatrick, J. D., \& Burgasser, A. J. 2007, AJ, 134, 1162

Monet, D. G., et al. 2003, AJ, 125, 984

Perryman, M. A. C., et al. 1997, A\&A, 323, L49

Reid, N., \& Hawley, S. L. 2000, New Light on Dark Stars: Red Dwarfs, Low Mass Stars, Brown Dwarfs (New York: Springer)

Reid, I. N., Lewitus, E., Burgasser, A. J., \& Cruz, K. L. 2006, ApJ, 639, 1114 Siegler, N., Close, L. M., Burgasser, A. J., Cruz, K. L., Marois, C., Macintosh, B., \& Barman, T. 2007, AJ, 133, 2320

Skrutskie, M. F., et al. 2006, AJ, 131, 1163

Stephens, D. C., \& Leggett, S. K. 2004, PASP, 116, 9

Tinney, C. G., Burgasser, A. J., \& Kirkpatrick, J. D. 2003, AJ, 126, 975

Tokunaga, A. T., Simons, D. A., \& Vacca, W. D. 2002, PASP, 114, 180

van Dam, M. A., et al. 2006, PASP, 118, 310

Vrba, F. J., et al. 2004, AJ, 127, 2948

Wizinowich, P. L., et al. 2006, Proc. SPIE, 6272, 7

Zapatero Osorio, M. R., Martín, E. L., Béjar, V. J. S., Bouy, H., Deshpande, R., \& Wainscoat, R. J. 2007, ApJ, 666, 1205 